\title{
Le Saint-Laurent, pivot du système défensif de Québec (1757-1759)
}

\section{Dave Noël}

\begin{abstract}
Anticipating the possible siege of Quebec, General Montcalm's staff started in 1757 to elaborate a defence system of the estuary of the St. Lawrence, a project which one can divide into two operational sectors: the Laurentian channel and the Quebec basin. In this article, the author examines the fundamentals of the French defensive system as well as the factors which contributed to its partial application in the spring of 1759.
\end{abstract}

« Je ne vois nulle part aucune armée qui se soit trouvée dans une position exactement semblable à la nôtre. Nous sommes assiégés par terre et par l'eau et nous sommes obligés de pourvoir à la défense d'une ville qui n'a pour toute ressource qu'une nombreuse artillerie. Une armée de terre nous observe, tandis qu'une flotte nous côtoye et influe par son approche à faire pencher la balance à notre désavantage. »

Officier français, Québec le 22 juillet $1759^{1}$

En 1759, le contrôle du Saint-Laurent par la flotte britannique assure la mobilité et la sécurité du corps expéditionnaire chargé de la prise de Québec. La domination navale des assiégeants a toutefois laissé dans l'ombre la stratégie élaborée par l'étatmajor de l'armée française dont l'ordre de bataille constitue un véritable système défensif de l'estuaire du Saint-Laurent; un projet que l'on peut diviser en deux secteurs opérationnels : le chenal laurentien et le bassin de Québec. Si la première partie du plan français n'a guère attiré l'attention des historiens en raison de son abandon, l'analyse de la seconde s'est quant à elle enlisée dans le débat opposant les partisans du gouverneur Pierre de Rigaud marquis de Vaudreuil, à ceux de Louis-Joseph de Montcalm. Personnifié par ce dernier, l'ordre de bataille adopté dans le bassin québécois est généralement associé à l'immobilisme, qu'ont perçu chez le général les historiens Frégault et Eccles². Largement répandue, leur appréciation ne tient toutefois pas compte des visées initiales du projet et des contraintes matérielles découlant de sa mise en oeuvre. Notre article vise

1 «Continuation du journal de la campagne de $1759 »$, Rapport de l'archiviste de la Province du Québec (1933-1934), p.100.

2 Guy Frégault, La guerre de la Conquête, Montréal, Fides, 1955, p.10, 315, 339. W.J. Eccles, «Louis-Joseph de Montcalm», Dictionnaire biographique du Canada, tome III, Québec, Presses de l'Université Laval, 1974, p.495-507.

The Northern Mariner/le marin du nord, XXI No. 1, (January 2011), 47-58 
donc à mettre en lumière les fondements de l'ensemble du système défensif de l'estuaire laurentien de même que les facteurs ayant contribué à la réduction de son périmètre fortifié au printemps de 1759 .

\section{La défense du chenal laurentien}

Wolfe : "Je vous avouerai naturellement que j'ai été très surpris d'avoir pu arriver avec la flotte angloise jusques visavis la ville de Québec sans avoir rencontré aucune chicanne dans le fleuve St-Laurent. "

Montcalm : "Ce n'est pas ma faute si vous n'avés pas trouvé des obstacles dans votre chemin (...) Je préparai le projet pour la partie du fleuve audessous de Québec; mais je ne commandai pas en chef pour pouvoir le faire exécuter. ${ }^{3} "$

Dans cet extrait du Dialogue des morts, le chevalier de Johnstone attribue au marquis de Montcalm, dont il a été l'aide-de-camp, la paternité d'un plan de défense de l'estuaire laurentien. Bien qu'il soit mentionné dans le cadre d'une discussion fictive, le projet évoqué par Johnstone se rapporte toutefois à un document bien réel: le mémoire intitulé Que peut faire de son côté la France pour la défense de Québec ${ }^{4}$. Soumis à Versailles en décembre 1758, ce projet constitue la refonte d'un plan conçu à l'automne de 1757 à partir d'un relevé détaillé du théâtre d'opérations de Québec. Cette année-là, l'officier d'artillerie Fiacre-François de Montbeillard est d'abord chargé d'évaluer les possibilités défensives offertes par la Côte-du-Sud, qu'il parcourt de la Pointe-Lévy à Kamouraska, avant de rejoindre Montcalm dans sa tournée d'inspection des côtes de Beauport et de Beaupré. Produit par l'état-major des troupes de terre, le mémoire rédigé à partir de ces relevés porte également la marque d'un navigateur émérite : Gabriel Pellegrin ${ }^{5}$.

Reconnu pour son expérience " pratique des mers d'Amérique et du fleuve SaintLaurent ${ }^{6} »$, Pellegrin réalise dès les années 1730 des levées hydrographiques dans le golfe et l'estuaire laurentiens avant d'être chargé du pilotage des vaisseaux de la marine royale du Bic jusqu'à la rade de Québec. En 1755, il dresse ses propres cartes du fleuve et procède à l'analyse critique de celles du bureau hydrographique de Paris ${ }^{7}$. Il est alors au sommet de sa carrière. Ayant fait la connaissance du marquis de Montcalm à Brest en

3 Chevalier de Johnstone, « Dialogue des morts », Literary and Historical Society of Quebec, Ninth series of historical documents, Québec, 1915, p.107-108.

4 Louis-Antoine de Bougainville, Écrits sur le Canada, Québec, Septentrion, 2003, p.28-31.

5 Pierre Pouchot, un capitaine du régiment de Béarn, s'attribue également la paternité de ce projet dans ses mémoires. Il s'agit toutefois d'une affirmation suspecte en raison de sa connaissance limitée de l'estuaire - il n'y est passé qu'une fois au printemps de 1755. Pierre Pouchot, Mémoires sur la dernière guerre de l'Amérique septentrionale, Québec, Septentrion, 2003, p.189.

6 H.-R. Casgrain (dir.), Collection des manuscrits du maréchal de Lévis, Montréal, Beauchemin, 1889-1895, v.7, Journal du marquis de Montcalm, p.49.

7 James S. Pritchard, " Gabriel Pellegrin », Dictionnaire Biographique du Canada, tome IV, Québec, Presses de l'Université Laval. Claude Boudreau, La cartographie au Québec: 1760-1840, Québec, Presses de l'Université Laval, 1994, p.32-33. 
1756, c'est au cours de leur traversée de l'Atlantique que Pellegrin initie le général aux principes de la navigation. Devenu son homme de confiance, il lui sert de guide l'année suivante lors de sa tournée d'inspection de l'estuaire et est recommandé à Versailles pour tout ce qui concerne la défense maritime de Québec. C'est à partir de leur association qu'est élaboré un système défensif visant à maximiser les difficultés inhérentes à la navigation laurentienne. Un tel projet, doit notamment provoquer une congestion à la tête de la flotte britannique, retardant du coup le début des opérations terrestres du siège de Québec. Ce faisant, les assiégés pourront profiter du climat de la colonie qui limite aux mois de mai à octobre la durée d'une campagne . $^{8}$

Le système de défense élaboré par l'état-major du général Montcalm en collaboration avec Gabriel Pellegrin comporte d'abord une partie terrestre basée sur un réseau de fortifications légères: les chicanes. Il s'agit d'un terme employé pour décrire des batteries de deux à quatre canons, ou mortiers, montés sur des plates-formes sommairement retranchées, que l'on érige de part et d'autre de la voie maritime du SaintLaurent, afin d'y concentrer leurs feux en direction de la flotte britannique ${ }^{9}$. Une première chicane est ainsi prévue dans la portion du chenal menant de l'île aux Coudres à l'île d'Orléans; un passage étroit et périlleux, dont l'entrée doit être défendue par deux batteries: l'une à la Pointe-aux-Bouleaux des Éboulements et la seconde à l'île aux Coudres, à proximité du mouillage de la Prairie ${ }^{10}$. Pour les auteurs du projet, ce havre « est nécessaire à tous vaisseaux et aucun bâtiment ne pourrait passer ${ }^{11}{ }$ ” en amont sans que les batteries chargées de sa défense ne soient forcées par un siège. C'est au mouillage de la Prairie, en effet, que l'on doit jeter l'ancre afin d'attendre le retour de la marée qui permet de s'engager plus en avant ${ }^{12}$.

Après avoir marqué un temps d'arrêt afin de sécuriser le goulet d'étranglement de l'île aux Coudres, l'avant-garde de la flotte britannique devra ensuite s'engager dans une seconde chicane aménagée à la traverse du cap Tourmente. C'est à cet endroit que

8 Le naufrage de deux navires dans le chenal sud de l'île d'Orléans à l'été de 1757 contribue notamment à l'élaboration de ce projet. Bougainville, un aide-de-camp de Montcalm, écrit alors que les écueils du Saint-Laurent et «sa navigation, la plus dangereuse et la plus difficile qu'il y ait, font le meilleur rempart de Québec ». Louis-Antoine de Bougainville, Écrits sur le Canada, p.242.

9 Bougainville se montre particulièrement favorable au principe des avant-postes retranchés. En 1758, ce membre influent de l'état-major suggère ainsi de retarder la remontée du lac Saint-Sacrement par les Britanniques à partir de l'île aux Moutons où il souhaite se retrancher avec quatre canons, 50 soldats et deux mois de vivres. Ibid., p.306-307.

10 En 1758, dans un rapport rédigé à partir d'observations tirées lors d'un voyage effectué six ans plus tôt, l'ingénieur Dubois propose lui aussi la défense du passage de l'île aux Coudres. Il met toutefois l'accent sur la batterie des Éboulements qui est un «poste inattaquable» en raison de l'escarpement et des broussailles qui s'y trouvent. Quant à l'île aux Coudres, Dubois propose d'y ériger une batterie près du mouillage de la Prairie ou de procéder à sa déforestation afin d'en faciliter le pilonnage à partir des Éboulements. Archives des Colonies, C11A, vol.104, p.398-403.

11 Louis-Antoine de Bougainville, Écrits sur le Canada, p.29.

12 Chevalier de Johnstone, « Dialogue des morts », p.108. 


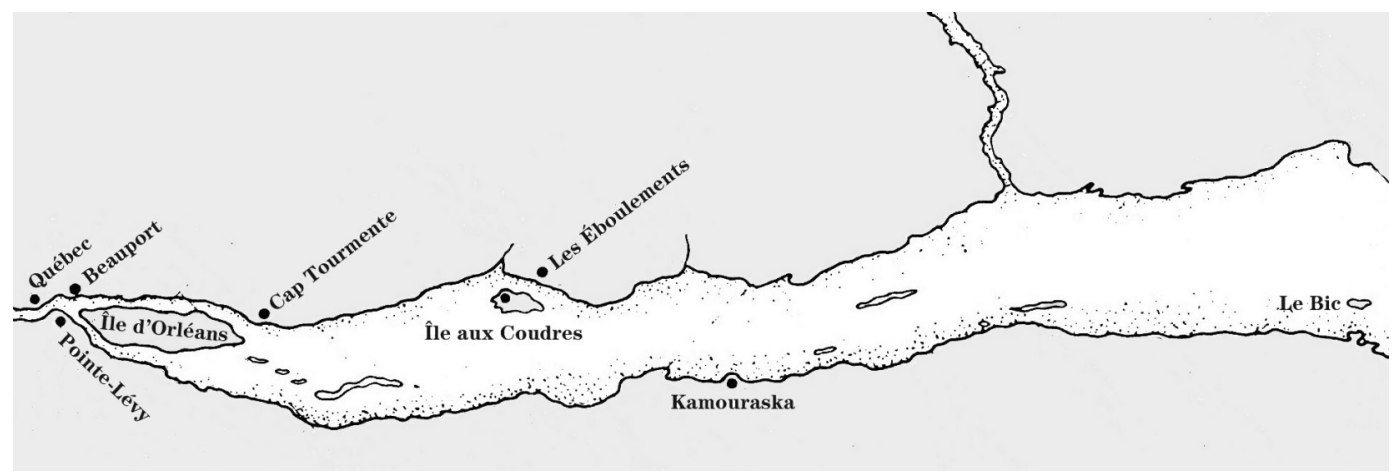

Carte 1: Chenal du Saint-Laurent. Infographie Amélie Tremblay.

les navires remontant le fleuve jusqu'à Québec bifurquent vers le chenal sud de l'île d'Orléans par une manœuvre qui les obligent à « ranger ce cap à portée de fusil et [à] lui présenter la poup $\mathrm{e}^{13} »$. Une batterie érigée à cet endroit permettrait ainsi un pilonnage intensif des bâtiments ennemis et ce, durant plus d'un quart d'heure. L'environnement hostile du cap Tourmente doit de plus favoriser le maintient de cette batterie tout au long de la campagne; les auteurs du projet ayant prévu détacher aux environs un corps de 150 miliciens et miquelets - des Catalans des Pyrénées spécialisés dans la guerre en terrain montagneux ${ }^{14}$. Quant à la sortie de la traverse, sa défense doit être assurée par une dernière chicane aménagée au mouillage de Saint-François, à la pointe nord-est de l'île d'Orléans. Bien que le mémoire remis à Versailles n'entre pas dans ces détails, une idée plus précise des travaux qui pourront être réalisés à cet endroit nous est donnée par Montbeillard qui recommande en 1758, la construction de batteries aux îles Madame et d'Orléans ${ }^{15}$.

Érigées rapidement et à peu de frais, les chicanes doivent êtres soutenues par une force mobile composée de vaisseaux de guerre. Déterminé par Gabriel Pellegrin, leur usage est détaillé dans une lettre approuvée par Montcalm qu'il remet au gouverneur Vaudreuil en janvier 1759. Deux alternatives sont alors formulées par le navigateur. Sur la base d'un renfort de 18 à 25 vaisseaux, Pellegrin propose d'abord la défense du bas estuaire à l'aide d'une croisière au large du Bic, un havre très « avantageux pour attendre l'ennemi qui, quand même il seroit de moitié plus fort, ne voudroit pas tenter une

13 Louis-Antoine de Bougainville, Écrits sur le Canada, p.29.

14 C'est au cours de la guerre de Succession d'Autriche, en Italie, que Montcalm est témoin de l'efficacité des miquelets. Réclamés avec insistance au sein des mémoires remis à Versailles, ces derniers ne sont toutefois jamais dépêchés dans la colonie. Louis-Antoine de Bougainville, Écrits sur le Canada, p.27, 29. Martin L. Nicolai, « A Different Kind of Courage: The French Military and the Canadian Irregular Soldier during the Seven Years' War », Canadian Historical Review, LXX, 1, 1989, p.53-75.

15 H.-R. Casgrain (dir.), Collection, v.4, Lettres et pièces militaires, p.73-74. Initialement, une batterie et un camp retranchés sont également prévus à Gaspé. Fondé sur la rumeur d'un établissement britannique à cet endroit, ce projet est toutefois rapidement abandonné. LouisAntoine de Bougainville, Écrits sur le Canada, p.28, 51-57. 
aventure ${ }^{16}{ }_{\text {}}$ en raison du corps expéditionnaire qu'il doit escorter. L'effet dissuasif d'une telle flotte peut alors s'appuyer sur l'exemple de Louisbourg, où la concentration d'une force similaire en 1757 a contribué au report du siège de la forteresse. Survolée par Pellegrin, cette hypothèse optimiste est suivie d'un ordre de bataille plus modeste, adapté à une escadre de 8 à 12 vaisseaux, une force que le navigateur souhaite diviser en deux parts : la première étant postée en réserve entre l'île d'Orléans et l'île aux Ruaux, et l'autre, en aval, à proximité de la batterie du cap Tourmente. L'avant-garde de cette force doit ainsi diriger un feu croisé en direction du chenal tout en conservant la possibilité de couper ses câbles afin de rejoindre, en combattant, le reste de son escadre. Quant au chenal nord de l'île d'Orléans, Pellegrin suggère d'en interdire l'accès à l'aide de trois navires ou frégates, appuyés par des bateaux armés à fond plat, capables de s'échouer sur les battures de la côte de Beaupré afin d'y servir de batteries flottantes ${ }^{17}$.

Misant sur un soutien raisonnable de la marine royale, le plan de défense du chenal laurentien n'est toutefois pas appliqué au printemps de 1759. Son abandon s'explique notamment par l'adoption à Versailles d'un projet de débarquement en Grande-Bretagne qui mobilise l'essentiel des vaisseaux français au détriment de Québec. Une escadre de huit vaisseaux est bien dépêchée en Amérique - au moment où les émissaires de la colonie sollicitent des renforts - mais c'est toutefois aux Caraïbes qu'elle est destinée. Elle y parvient d'ailleurs trop tard pour soutenir la Guadeloupe qui tombe aux mains des Britanniques. Repliée à Saint-Domingue, cette escadre en repart le 18 septembre pour la France, soit le jour même de la capitulation de Québec ${ }^{18}$. Déjà compromis par les choix tactiques des autorités françaises, le projet de défense du chenal laurentien doit être définitivement abandonné suite à l'arrivée tardive dans la capitale de l'état-major du marquis de Montcalm. Retenu à Montréal par le gouverneur Vaudreuil, ce n'est que dans la nuit du 22 mai que le général gagne enfin le théâtre d'opérations dont il doit préparer la défense. Six jours plus tard, l'avant-garde de la flotte britannique a déjà pris le contrôle du passage de l'île aux Coudres.

En dépit de son abandon au printemps de 1759, la partie du chenal laurentien située en aval de Québec fait tout de même l'objet d'une série de mesures défensives. Au cours des semaines précédant le siège, un navire est dépêché vers Mont-Louis afin de «ramasser les pilotes de la rivière répandus le long de ses $\operatorname{côtes}^{19}{ }^{19}$; les balises de

16 H.-R. Casgrain (dir.), Collection, v.4, Lettres et pièces militaires, p.91-92.

17 Ibid., p.94.

18 Christian Buchet, La lutte pour l'espace caraïbe et la façade atlantique de l'Amérique Centrale et du Sud (1672-1763), Paris, Librairie de l'Inde, 1991, p.396. Jonathan R. Dull, La guerre de Sept Ans : histoire navale, politique et diplomatique, Bécherel, Les Perséides, 2009 , p.180.

19 Armé par le munitionnaire Cadet, ce navire est intercepté avant d'atteindre sa destination. Il s'agit toutefois d'une perte sans conséquence, les Britanniques s'étant déjà assuré le concours de pilotes canadiens et français capturés lors des campagnes précédentes. «Attestation de M. le marquis de Vaudreuil », 28 septembre 1767, A.G. Doughty et G.W. Parmelee, The Siege of Quebec and the Battle of the Plains of Abraham, Québec, Dussault et Proulx, 1901, v.5, p.359. À son arrivée au mouillage de la Prairie, l'avant-garde de la flotte britannique aurait également arboré le drapeau français afin de capturer les pilotes de l'île 
l'estuaire y sont remplacées par de fausses bouées tandis que sont détruits sur l'île d'Orléans et l'île aux Ruaux les repères visuels utilisés par les pilotes engagés dans la traverse $^{20}$. À l'île aux Coudres, un officier doit également lancer des radeaux chargés de combustibles en direction des navires britanniques attendus au mouillage de la Prairie; des brûlots qui devront toutefois être détruits sur place, faute de temps pour les armer. Au cap Tourmente, Gabriel Pellegrin reçoit enfin le mandat d'évaluer la possibilité d'obstruer la traverse par le sabord d'une dizaine de bâtiments. Le chenal étant toutefois jugé trop large pour ce type d'opération, on propose d'y positionner quelques frégates afin de les opposer aux barges britanniques chargées de le sonder. Divisé quant à l'usage de sa marine, le conseil de guerre présidé par Vaudreuil va trancher : deux des huit frégates disponibles iront mouiller au-dessus de Québec, à l'Anse-des-Mères, tandis que le reste sera partiellement désarmé afin de rejoindre le dépôt de vivres établi à la rivière Batiscan $^{21}$.

Quant à l'île d'Orléans, qui n'a pas fait l'objet de la tournée d'inspection de 1757, on y dépêche d'abord un ingénieur afin « d'y trouver un terrain avantageux pour y établir quelques batteries ${ }^{22} \gg$. L'ampleur des travaux nécessaires afin de couvrir ce type d'ouvrage dans une île qui n'est «protégée de rien et d'un facile accès » provoque toutefois l'abandon du projet, une situation anticipée par Montbeillard qui estime qu'il « falloit aider la nature à ses points essentiels; mais s'y prendre ${ }^{23}$ » deux ans plus tôt. À la mi-juin, une batterie est tout de même érigée à Saint-François afin de tirer à boulets rouges sur l'avant-garde de la flotte britannique. L'artillerie de l'île d'Orléans en est toutefois retirée dès le 18 juin tandis que le corps d'armée détaché aux environs se replie le 26. Le lendemain, c'est toute l'armée du général James Wolfe qui débarque à l'île d'Orléans, ce qui marque le début des opérations du siège de Québec.

\section{La défense du bassin québécois}

Privé du soutien naval escompté, l'état-major de Montcalm doit se replier sur la seconde partie du système de défense de l'estuaire laurentien. Axé sur la fortification de la rive gauche du bassin de Québec, le périmètre de ce projet a déjà été circonscrit dans la colonie. En effet, dès 1745, en se basant sur l'exemple du siège de Carthagène, l'ingénieur Chaussegros de Léry propose une défense de Québec à partir des battures de Beauport $^{24}$. Dix ans plus tard, c'est au tour de l'officier d'artillerie François Le Mercier

aux Coudres. John Knox, The Siege of Quebec and the Campaigns in North America 17571760, Mississauga, Pendragon House, 1980, p.123.

20

21

Archives des Colonies, C11A, vol.104, p.72.

H.-R. Casgrain (dir.), Collection, v.7, Journal du marquis de Montcalm, p.533.

"Journal du siège de Québec du 10 mai au 18 septembre 1758 », annoté par Aegidius Fauteux, Rapport de l'archiviste de la Province de Québec (1920-1921), p.141.

H.-R. Casgrain (dir.), Collection, v.7, Journal du marquis de Montcalm, p.551.

Léry tire également profit de l'expérience des vétérans de la descente de Camaret en Bretagne (1694) au cours de laquelle les Anglais ont été repoussés dans leurs chaloupes de débarquement. Pierre-Georges Roy (dir.), Inventaire des Papiers de Léry, 2 vol., Québec, 1939, v.2, p.55. Au sein du corps d'armée britannique présent au siège de Carthagène de 
d'évoquer la ligne fortifiée devant être érigée entre les rivières Saint-Charles et Montmorency ${ }^{25}$. Intégré au plan de 1757, ce projet est conservé l'année suivante au sein du mémoire soumis à Versailles. Mis en œuvre par Montcalm au printemps de 1759, les travaux nécessaires à la réalisation de ce plan sont ensuite approuvés par le gouverneur Vaudreuil. Réduit à la rive gauche du bassin de Québec, le système défensif de la capitale doit initialement s'étendre jusqu'à la Pointe-Lévy où une batterie est prévue au cap Samson à la sortie du chenal orléanais ${ }^{26}$. Demeurée sans défense en juin 1759 , la rive droite du bassin est alors occupée par les Britanniques dont les batteries, érigées sur les hauteurs, favorisent le passage de leurs navires en amont de la capitale. Largement critiqué, l'abandon de la Pointe-Lévy aux assiégeants est généralement présenté sous l'angle d'une mauvaise évaluation du théâtre d'opérations de Québec. Une telle décision s'explique toutefois par le court laps de temps alloué à la fortification des parties essentielles du front laurentien. Sous-estimée, cette contrainte mérite que l'on s'y attarde afin de mieux cerner la partie appliquée du système de défense du Saint-Laurent.

Au printemps de 1759, c'est en l'espace de cinq semaines seulement que la rive gauche du bassin de Québec doit être aménagée afin de recevoir les assiégeants ${ }^{27}$. Il s'agit là de travaux élémentaires qui visent à offrir des points d'appui à l'armée plutôt qu'un front étanche et statique. Cette nuance, Montcalm doit d'ailleurs la rappeler à ses propres soldats qui, demeurés marqués par la bataille de Carillon, réclament des retranchements supplémentaires pour se couvrir ${ }^{28}$. Or, ces derniers sont prévenus qu'en dehors d'une attaque à marée haute, la ligne de Beauport ne doit leur servir d'abri que lors du barrage d'artillerie précédant une descente britannique. Ainsi, dans l'éventualité d'un débarquement à marée basse ou à demi-marée, l'essentiel sera « de tomber brusquement sur l'ennemi » afin de le culbuter dans ses barges ${ }^{29}$. L'importance accordée à cette manœuvre est matérialisée par l'installation d'une série de poteaux le long des sentiers menant du chemin de Beauport au bassin afin d'y assurer une concentration rapide des

1741, se trouve James Murray, le troisième brigadier du général Wolfe, de même que le père de ce dernier, le major-général Edward Wolfe.

H.-R. Casgrain (dir.), Extraits des archives des ministères de la Marine et de la Guerre à Paris, 3 vol., Québec, Demers et Frères, 1890, v.1, p.155-156.

26 Louis-Antoine de Bougainville, Écrits sur le Canada, p.29. H.-R. Casgrain (dir.), Collection, v.4, Lettres et pièces militaires, p.64. Selon Knox, Montcalm aurait souhaité établir 4000 hommes dans un camp retranché à la Pointe-Lévy, une information qu'il dit tenir d'officiers français rencontrés après la prise de Québec. John Knox, The Siege of Quebec, p.138.

27 Le système défensif de Québec est conçu en prévision d'un assiégeant de la taille de l'armée britannique présente au siège de Louisbourg de 1758, un estimé qui concorde avec les 12000 hommes initialement promis au général Wolfe dont les effectifs sont finalement réduits à 8635 soldats. Leicester Harmsworth (dir.), Collection Northcliffe, Ottawa, F. A. Acland, 1927, p. 142 .

28 Le 31 mai 1759, Montcalm rappelle à ce sujet qu'il ne faut « pas faire des travaux inutiles, qui ne sauroient être achevés, qui fatigueroient trop le soldat, qui ne sauroient être gardés, ou, s'ils l'étoient, affoiblieroient trop l'armée ». H.-R. Casgrain (dir.), Collection, v.4, Lettres et pièces militaires, p.168.

29 Livre d'ordres de l'armée du marquis de Montcalm et du chevalier de Lévis, ZQ7, vol. 4 : Campagne de 1759, p.64-65, 71. 
troupes dans l'obscurité ${ }^{30}$. On veut ainsi éviter la répétition du siège de Louisbourg de 1758 au cours duquel l'armée française s'est montrée incapable de repousser promptement les Britanniques débarqués dans la baie de Gabarus ${ }^{31}$.

Basé sur une défense côtière souple et dynamique, l'ordre de bataille de Montcalm nécessite néanmoins la mise en œuvre d'un vaste chantier naval et terrestre. Mais auparavant, des travaux doivent être réalisés dans Québec dont l'enceinte est complétée par des palissades érigées sur le pourtour du Cap-Diamant. Des barricades sont également aménagées entre la haute et la basse-ville tandis que sont maçonnés les rez-dechaussée des habitations donnant sur le fleuve. Des hangars et des toitures sont démontés pour prévenir les incendies et des batteries sont érigées ou reconstruites afin d'assurer la sécurité des artilleurs. Ainsi, bien que la fonction défensive de Québec soit limitée - la ville faisant office de grosse redoute à la droite de Beauport - sa mise en état engloutit du temps et des matériaux; ce qui amène plusieurs officiers à proposer la destruction de la basse-ville, dont Montbeillard, qui constate qu'il « est plus aisé de construire à neuf que de réparer du vieux ${ }^{32} \gg$. L'artilleur fait ici référence aux travaux en cour dans les campagnes environnantes. Le long de la rivière Saint-Charles notamment, dont la rive droite est retranchée du faubourg Saint-Roch à 1'Hôpital-Général. Fermé à son embouchure par une estacade, ce cours d'eau doit servir de seconde ligne au front de Beauport dont la côte est hérissée de batteries et de redoutes. À ces ouvrages s'ajoutent différents travaux de voirie visant à assurer la mobilité de l'armée et son ravitaillement. Des ponts sont jetés à cette fin sur les rivières Saint-Charles, Cap-Rouge et JacquesCartier tandis que de nouvelles routes sont tracées dans la vallée de la Saint-Charles.

Mené en parallèle, un chantier naval est réalisé dans le bassin de Québec où l'on

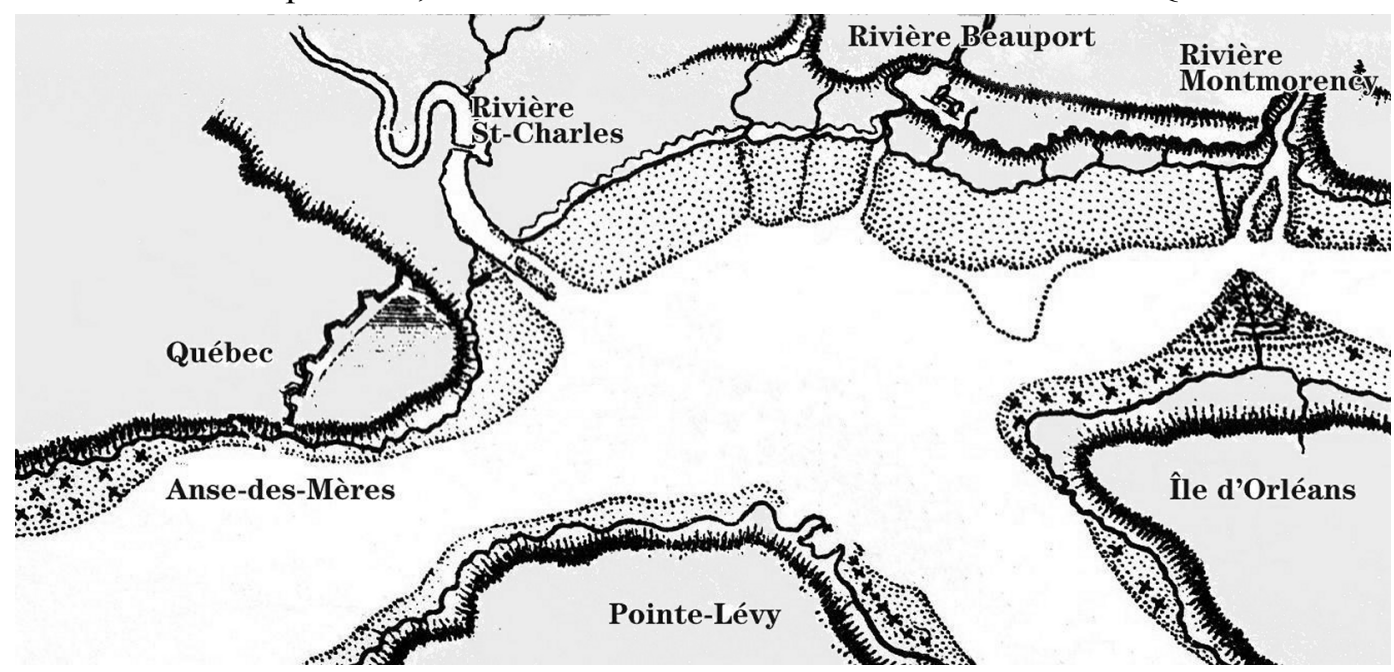

Carte 2 : Bassin de Québec. D'après A New Chart of the River St. Lawrence. Thomas Jefferys, 1775, BAC. Infographie Amélie Tremblay.

30 Ibid., p.77, 82.

31 Louis-Antoine de Bougainville, Écrits sur le Canada, p.267.

32 H.-R. Casgrain (dir.), Collection, v.7, Journal du marquis de Montcalm, p.539. 
dépêche Gabriel Pellegrin afin de vérifier «à quelle distance peuvent mouiller les vaisseaux dans toute la longueur de la plage de Beauport». Estimée à 700 toises pour une frégate et à 900 pour un vaisseau, c'est dans cet espace parsemé d'écueils que pourra manœuvrer la flottille côtière que l'on met en chantier ${ }^{33}$, une force navale composée d'un transport armé, de douze gros canots - chacun monté d'un canon léger - et d'une demidouzaine de chaloupes armées de canons lourds. N'offrant qu'une faible surface de tir à l'artillerie britannique, ces bâtiments sont conçus pour couler une barge et soutenir le feu d'une frégate ${ }^{34}$. Guidés par des canots d'écorces, ils doivent patrouiller le bassin de Québec et servir de première ligne à la côte de Beauport où ils bénéficient du soutien de trois batteries flottantes. Les deux premières, fabriquées à partir de navires marchands démâtés, sont échouées à l'entrée de la rivière Saint-Charles. Quant à la troisième, manœuvrant à rames et à voiles, elle est spécialement conçue pour la défense de la rivière Beauport. À ces bâtiments s'ajoute une flottille de brûlots composée de huit navires et de 120 radeaux enchaînés les uns aux autres. Des prototypes sont également construits, dont un canot à caissons semi-submersibles et un radeau chargé de fusils et de ressorts qui doit exploser au contact du premier bâtiment rencontré dans le bassin ${ }^{35}$.

Au cours des semaines précédant le siège, la mise en œuvre de l'ordre de bataille élaboré par l'état-major de Montcalm est entravé par de nombreux obstacles. Dans un premier temps, l'ampleur du chantier à réaliser provoque un certain flottement au sein du camp français, ce qu'observe Montbeillard lorsqu'il écrit que « dans la crainte de ne pas finir [les travaux] on ne commence rien ${ }^{36} »$. Les délais engendrés par cette torpeur sont aggravés par les pluies diluviennes et les grandes marées du printemps, qui emportent une partie des ouvrages, érigés trop près des battures. Aux intempéries s'ajoute une pénurie d'outils et de bois que l'on fait venir de l'arrière-pays de Beauport afin de préserver les

33 Ibid, p.535. Les mesures de Pellegrin vont s'avérer justes. Dans son bilan de campagne, le général Wolfe écrit à cet effet: «As the men of war cannot, for want of a sufficient depth of water, come near enough to the enemy's intrenchments, to annoy them ». "Wolfe's Dispatch to Pitt, 2 September 1759 », dans C.P. Stacey, Quebec, 1759. The Siege and the Battle, texte édité par Donald. E. Graves, Toronto, Robin Brass Studio, 2002, p.195.

34 Les canots armés sont qualifiés de jacobites du nom de leur inventeur canadien LouisThomas Jacau de Fiedmont. Souvent confondus avec les chaloupes-canonnières (carcassières), les jacobites sont conçus à partir de « bateau de roy » pontés, dont l'avant est égueulé afin de permettre un tir frontal. Nicolas Renaud d'Avène des Méloizes, Journal militaire 1756-1759, Québec, 1930, p.38. Le $1^{\text {er }}$ juillet, une partie de cette flottille est également utilisée afin de bombarder les Britanniques établis à la Pointe-Lévy.

35 Le cajeu à ressorts est composée de quatre étages de grenades et de fusils devant foudroyer les matelots chargés de le détourner. Jean-Félix Récher, Journal du siège de Québec en 1759, Québec, Société historique de Québec, 1959, p.32. Quant au cajeu semi-submersible, il est construit à partir d'un « canot de 8 à 9 pieds de long, pointu par un bout, et séparé en trois parties »; les deux extrémités devant contenir les explosifs et les marins tandis que l'espace mitoyen doit «se remplir d'eau afin de pouvoir se caler et raser le dessus " du bassin de Québec. Louis-Léonard Aumasson de Courville, Mémoires sur le Canada depuis 1749 jusqu'à 1760 en trois parties, avec cartes et plans lithographiés. Québec, Société littéraire et historique de Québec, Québec, Middleton et Dawson, p.143. 
broussailles et les clôtures qui facilitent la défense de la côte. Un manque de maind'œuvre frappe enfin le chantier en raison de l'envoi au lac Champlain, au début du mois de mai, d'une partie des milices du Gouvernement de Québec et des bataillons cantonnés aux environs, une manœuvre vivement regrettée à l'annonce d'une flotte britannique dans l'estuaire. Un témoin raconte que l'on sentit alors à Québec « le tort qu'on avait eu d'en faire partir les trois bataillons » puisque « le temps qu'il fallait mettre à en faire descendre d'autres, parut un temps précieux, et qui était perdu ${ }^{37} \gg$. Quant aux milices destinées à la fortification de la capitale, leur mobilisation est toujours en cours au moment où les Britanniques débarquent à l'île d'Orléans; les retranchements de Beauport devront ainsi être complétés au milieu des opérations militaires.

À l'origine, le système défensif de Québec doit être contenu dans les limites de son bassin. C'est pourquoi deux frégates sont d'abord mouillées à l'Anse-des-Mères où elles sont chargées d'intercepter les navires britanniques à l'aide du support des batteries de la basse-ville ${ }^{38}$. Proposée par Montcalm au conseil de guerre du 31 mai, cette mesure est maintenue le 10 juin au sein de l'ordre de bataille définitif rédigé par le chevalier de Lévis $^{39}$. Onze jours plus tard, un contrordre du gouverneur Vaudreuil provoque toutefois le repli des frégates au rapide du Richelieu, près de Deschambault, une décision qui favorise, en juillet, le passage du détroit de Québec par le Sutherland, un vaisseau qui fait «l'effet d'une citadelle» auprès des barges britanniques déployées en amont ${ }^{40}$. Réagissant à cette manœuvre, les assiégés dépêchent une partie de leur flottille côtière audessus de Québec où elle gêne considérablement les communications et les tentatives de

37 Jean-Claude Hébert (dir.), Le siège de Québec en 1759 par trois témoins, Québec, Ministère des affaires culturelles, 1972, p.54-55. Motivée par une pénurie de vivres, cette manœuvre est désapprouvé par Montcalm qui estime en mars 1759 qu'il faut « tirer le moins d'hommes qu'il se pourra de ce gouvernement » afin d'amorcer rapidement la défense de Québec. Un avis que ne partage pas Vaudreuil qui ne voit « aucun inconvénient à tirer des habitants de ce gouvernement, étant assuré de les faire descendre promptement au besoin ». H.-R. Casgrain (dir.), Collection, v.4, Lettres et pièces militaires, p.150-151.

38 Un témoin observe à ce sujet que « les entreprises heureuses de l'ennemi étaient dues au parti qu'on avait pris d'abandonner la rivière [...] contre l'opinion de quelques gens sûrs, qui avaient soutenu qu'il fallait tenir les deux frégates du roi bien armées au-dessus de Québec; lesquelles auraient empêché les berges anglaises de paraître de ce côté-là, et nous auraient assuré la navigation de la rivière. Les vaisseaux anglais n'eurent pu les en déloger, attendu qu'ils ne pouvaient remonter qu'en faisant sonder devant eux, ce que les frégates n'auraient point souffert; ou bien, si au risque de se perdre, ils avaient voulu forcer sur elles, il leur était facile de céder le terrain et de le faire toujours à propos ». Jean-Claude Hébert (dir.), Le siège de Québec en 1759, p.106.

39 Dans son «Mémoire sur la défense de Québec » du 31 mai 1759, Montcalm « estime que deux [frégates] au-dessus de Québec, dans la partie de Sillery, feront très bien ». H.-R. Casgrain (dir.), Collection, v.4, Lettres et pièces militaires, p.170. Quant au chevalier de Lévis, il note le 10 juin qu'il «n'y a pas lieu de croire que les ennemis pensent à tenter à passer devant la ville et à faire leur débarquement à l'anse des mers, et tant que les frégates subsisteront nous n'avons du moins rien à craindre pour cette partie ». Leicester Harmsworth (dir.), Collection Northcliffe, p.226.

40 Jean-Claude Hébert (dir.), Le siège de Québec en 1759, p.90. 
débarquement du brigadier James Murray ${ }^{41}$. De petits postes fortifiés sont également établis le long de la rive nord du Saint-Laurent jusqu'à l'embouchure de la rivière Jacques-Cartier, une extension de la ligne française qui provoque l'essoufflement des troupes, dont la fatigue est aggravée par un rationnement sévère à partir du mois d'août. À cela s'ajoute une diminution notable des effectifs par la désertion des milices, le départ des alliés amérindiens et l'envoi de renforts vers Montréal. Quant aux Britanniques, bien que décimés par la maladie et les combats, leur force de frappe demeure intacte tout au long du siège. En effet, comme l'observe Johnstone dans son Dialogue: " celui qui attaque a toute sa force réunie et concentrée dans un seul point » tandis que « celui qui l'attend a sa force divisée sur toute l'étendue de ses lignes ${ }^{42} \gg$; une maxime appliquée par le général Wolfe dont la manœuvre du 13 septembre 1759 force Montcalm à sortir de son système défensif afin de lui livrer une bataille risquée, mais nécessaire, dont le résultat mène à la chute de Québec.

\section{Conclusion}

La mise en œuvre de la première partie du système défensif de l'estuaire laurentien aurait-elle permis de retarder la remontée du fleuve par la flotte britannique? Avec le recul, le brigadier d'infanterie Bourlamaque répond par la négative à cette question en soutenant que le «St-Laurent n'offre aucune défense jusqu'à Québec » et ce, bien qu'il ait été « dit souvent qu'il y avait des îles ou des caps propres à barrer le cours $\mathrm{du}$ fleuve ${ }^{43} \gg$. Cet avis est toutefois contredit par l'officier d'artillerie Montbeillard qui juge que les Britanniques " ne seroient pas venus avec autant de sécurité » si des chicanes avaient été mises en place à l'île aux Coudres et à la traverse $e^{44}$. En tenant compte du temps consacré au sondage du chenal par le capitaine William Gordon et de la prudence manifestée par son supérieur, le contre-amiral Philip Durell, il appert que la réalisation de ce projet aurait permis un report significatif des opérations terrestres du siège de Québec, favorisant du coup la consolidation des ouvrages prévus au sein de la seconde partie du système défensif de la capitale.

Quant à l'ordre de bataille adopté dans le bassin québécois, il comporte évidemment des failles, ses concepteurs ne possédant pas l'expertise amphibie développée par leurs homologues britanniques. Au cours du printemps de 1759, les membres de l'état-major du marquis de Montcalm ont toutefois mis à profit leur connaissance de la géographie locale de même que leur expérience navale, acquise au cours des campagnes précédentes, le long des lacs Ontario et Saint-Sacrement. Ils ont de plus manifesté une ouverture d'esprit étonnante envers les innovations technologiques proposées par de simples matelots ou marins autodidactes. C'est ce qui les a conduit à la désastreuse opération des brûlots, mais également à la conception d'une flottille côtière

41 Stephen Brumwell, Paths of Glory: The Life and Death of General James Wolfe, Montreal \& Kingston, McGill-Queen's University Press, 2006, p.236

42 Chevalier de Johnstone, « Dialogue des morts », p.111.

43 François-Charles de Bourlamaque, «Mémoire sur le Canada», Bulletin des Recherches Historiques, 26, 7, (juillet 1920), p.201-202.

44 H.-R. Casgrain (dir.), Collection, v.7, Journal du marquis de Montcalm, p.538-539. 
dont l'efficacité est reconnue par le général Wolfe lui-même. Dix jours avant sa mort, le commandant britannique écrit à ce sujet que : « to the uncommon strength of the country, the enemy have added, for the defence of the river, a great number of floating batteries and boats. By the vigilance of these (...) it has been impossible to execute any thing by surprize $^{45} \gg$. Ce constat d'échec met en relief l'efficacité d'un système défensif adapté à la singularité d'un fleuve faisant à la fois office de rempart et de champ de bataille.

45 «Wolfe's Dispatch to Pitt, 2 September 1759 », Stacey, Quebec, 1759, p.197. 